\begin{tabular}{|l|l|}
$\begin{array}{l}\text { Postprint } \\
\text { Version }\end{array}$ & 1.0 \\
\hline Journal website & http://www.sciencedirect.com/science/article/pii/S0266613812002501 \\
\hline Pubmed link & $\underline{\text { http://www.ncbi.nlm.nih.gov/pubmed/23427853 }}$ \\
\hline DOI & $10.1016 /$ j.midw.2012.12.009
\end{tabular}

This is a NIVEL certified Post Print, more info at http://www.nivel.eu

\title{
Organisation of emergency transfer in maternity care in the Netherlands
}

\author{
Therese A. Wiegers, PhD (PSyChologist, EPIDEMiologist, SENiOR RESEARCHER), \\ JULIA DE BORST, MA (JUNIOR RESEARCHER, ADVISOR IN HEALTH CARE AT KPMG PLEXUS) \\ NIVEL (Netherlands Institute for Health Services Research), P.O. Box 1568, 3500 BN \\ Utrecht, The Netherlands
}

\begin{abstract}
Objective: to gain more insight in the perceptions and experiences of care providers and clients with the organisation of emergency transfer in maternity care, with regard to transportation, responsibilities and communication between caregivers.

Background: in the Netherlands a woman with an uncomplicated pregnancy can choose to give birth at home, assisted by her own midwife. However, when complications arise, she needs to be referred to a hospital. In case of an emergency this referral must be effectuated quickly, often with ambulance support.

Design: a mixed methods' study consisting of semi-structured interviews and surveys.

Methods: qualitative data of the current organisation of emergency transfer in maternity care, including experiences of caregivers were collected through 21 semi-structured interviews. On the basis of the qualitative data questionnaires for a survey were developed. These questionnaires were sent to 181 caregiver organisations and an unknown number of clients. The response among caregivers was 108 (60\%), the response among clients was 42 .

Findings: the overall result from the interviews as well as from the questionnaires is that at the personal level cooperation is often adequate, but mostly informal. Care providers from different professions explained in the study that in emergency situations they usually know how to find each other but they are not always aware of existing agreements or protocols, are unsure of each other's competencies and do not always know what to expect from other care providers. Looking back at their experiences the majority of the clients responded that they were taken very good care of at the hospital.

Key conclusion and implications for practice: because transfer of care from one care provider to another during labour or birth is not unusual in the Dutch
\end{abstract}


maternity care system it is necessary for care providers involved in this transfer to know and trust each other, to be able to give an adequate reaction when needed. Recommendations given are to devise a protocol for midwives when calling an ambulance dispatch centre, to improve the knowledge about each other, for instance by providing combined courses for emergency obstetrics, preferably at the regional level, so people who actually may work together can train together, and to improve the referral process from midwife to obstetrician.

\section{INTRODUCTION}

In the Netherlands a home birth, assisted by a midwife, is still common, although numbers are declining. In 2008, the proportion of women giving birth in hospital under supervision of an obstetrician was 67 per cent, whereas 23 per cent gave birth at home and 10 per cent gave birth in a birth centre or hospital ward, accompanied by their own midwife (PRN, 2011). When complications arise during a home birth or in a birth centre, the woman needs to be referred to the hospital. In case of an emergency this referral must be effectuated quickly, often with ambulance support. This referral and transfer from community midwife or GP (primary care) to gynaecologist/obstetrician (secondary care) has recently been put in the centre of the debate about quality and organisation of maternity care in the Netherlands. The reason for this was the second Peristat publication indicating that the perinatal mortality rate in the Netherlands is higher than in most other European countries (EURO-PeRISTAT, 2008). Because there are no simple explanations for the differences between countries (Evers et al., 2010), attention was drawn to the organisation of maternity care, which in the Netherlands differs profoundly from that in all other European countries.

In the Netherlands midwives are independent medical practitioners who provide full maternity care, that is: antenatal, natal and postnatal care, to women with a low-risk status. Midwives are responsible for the initial risk-selection according to the Obstetric Manual, a list of (medical) indications and complications with recommendations for the appropriate level of care, agreed upon by all three professional organisations (midwives, GPs, and obstetricians) (CVZ, 2003). As risk factors can arise at any time, risk-selection is a continuous process and referral can take place at any moment during pregnancy, labour, birth or postpartum period. There are 91 general hospitals in the Netherlands, of which eight are University Medical Centres (UMCs). Most of the hospitals have labour wards where a midwife can refer her client to in case of (threatening) complications. The organisation of trauma care, including emergency obstetrics, is coordinated in 11 the so-called 'trauma regions' with a UMC or other large hospital as trauma centre. There are 25 ambulance dispatch centres in the Netherlands with almost 700 ambulances, stationed at more than 200 locations (Ambulancezorg Nederland, 2010).

Most pregnant women are healthy with no known risk factors and they start their antenatal care with a midwife. About fifteen per cent start antenatal care with an obstetrician because their risk profile cannot be considered to be low, based on their medical or obstetric history, and they stay in secondary care until after giving birth. An estimated 36 per cent of all pregnant women experience referral from a primary care midwife to a secondary care obstetrician early or late in pregnancy, but before the onset of labour. About 15 per cent of all pregnant women or $31 \%$ of women in 
midwifery care at the onset of labour, experience referral during or immediately after labour and birth (CBS, 2009 and PRN, 2011). Fig. 1 shows the referral rates and the actual place of birth in 2008.

\section{[FIGURE 1]}

Referrals before the onset of labour are seldom emergencies that need immediate interventions. They rather lead to a change in the consultation schedule, to include one or more visits to an obstetrician. Referrals during labour, especially when a birth at home or in a birth centre has been planned, involve unplanned and sometimes emergency transfer to a hospital. There is very little information available on the frequency of emergency transfers for pregnant or labouring women, because 'emergency' or 'urgency' is not included in the usual registrations. However, by studying the reasons for referral a distinction can be made between urgent and not urgent. Amelink-Verburg et al. (2008) showed that 31.9 per cent of the women who started labour under the care of a midwife in the years 2001-2003 were referred to secondary care, $28.3 \%$ without and $3.6 \%$ with urgency. Of the women who had planned to give birth at home $3.4 \%$ experienced an urgent referral, while that was the case for $4.1 \%$ of the women who planned to give birth in hospital, assisted by their own midwife. Converting this per centage to the total number of births in the Netherlands in 2011 means that each year about 3000 women experience an urgent referral during labour or birth, about 2000 of whom have planned a home birth and thus need transport.

There are also very few data available about emergency transfers from home to hospital in other developed countries. Johnson and Daviss (2001) reported that approximately $2 \%$ of clients of midwives who practice out-of hospital in 2000 in the US were transported for potentially life-threatening emergencies and approximately $6 \%$ for precautionary reasons. A later publication provided more detailed information albeit with a different definition: $12.1 \%$ of women intending to give birth at home with a certified professional midwife in the US were transferred to hospital intrapartum or post partum, with 3.4\% needing urgent transport (2.1\% before and $1.3 \%$ after birth of the infant) (Johnson and Daviss, 2005). In addition, transport stories have been published as indication of 'how reproduction can go unnecessarily awry when domains of knowledge conflict' (Davis-Floyd, 2003). Davis-Floyd summarises several ways in which this can occur. For instance: the midwife waiting too long to summon transport, the hospital staff not understanding the urgency of the problems, the ambulance team failing to respond properly, or miscommunication between midwife and ambulance team or midwife and hospital staff. Although the situation of home birth midwives in the USA and Mexico is very different from that in the Netherlands, some similar critical issues or events or circumstances that may hamper the cooperation between professionals in obstetric emergency transport may be found.

This exploratory study investigates the perceptions and experiences of care providers and clients with the organisation of emergency transfer of pregnant or labouring women in the Netherlands. The aim of this study is to gain more insight into the way 
emergency transfers in maternity care are organised, with regard to transportation, responsibilities, communication and cooperation between care providers, the bottlenecks or critical issues, events and circumstances, and the potential for improvement.

\section{METHODS}

\section{Study design}

This paper reports on a study consisting of semi-structured interviews followed by surveys. Information about the organisation of emergency transfer in maternity care has been collected through interviews. The themes resulting from the interviews and from literature were used in a survey among professionals and clients in order to confirm and quantify these perceptions and experiences. The purpose of including clients was to see emergency transfers from the clients' perspective, and to learn from their experiences, not to arrive at conclusions about clients in general.

\section{Sampling}

Interview candidates were selected on the basis of their profession and the trauma region they were working in and contacted through their professional organisations (purposive sampling). The aim was to interview at least one professional from each trauma-region and at least four from each position in the 'chain-of-care': primary care midwives, general practitioners, ambulance personnel and labour ward staff. Questionnaires were sent to 180 care provider organisations: 88 midwifery practices, 44 hospitals with labour wards, 25 ambulance services and 23 GP out-of-hours cooperatives, with the request that one of the persons most knowledgeable about emergency transfer would respond. The samples of midwifery practices and hospitals were stratified by the trauma region. All ambulance services and all GP out-of-hours cooperatives were included in the sample. In addition, the ambulance services were asked to provide registration data about the number of maternity care clients they were called in for in the previous year. Finally, the midwifery practices in the sample were all asked to hand out questionnaires to clients who had experienced an emergency situation during a period of six months and 45 of them agreed to do so. Each of these 45 practices received five copies of the client questionnaire to hand out, but did not register the number of questionnaires they actually handed out to clients. To ensure anonymity clients could return the questionnaire with a stamped, addressed envelope directly to the researchers. The clients were asked to describe the emergency situation they experienced.

\section{Instruments}

The interview topic list was based on available literature (Amelink-Verburg et al., 2008) and policy and research reports (Meiberg et al., 2007; Offferhaus and van der Hoff, 2008). The interview was furthermore structured according to the chain of events: the chronological order of what happens when an emergency arises. Each interview started with an open question about personal experiences with emergency transfer in maternity care, followed by questions about available guidelines or protocols, cooperation between care providers, bottlenecks or critical issues, and an evaluation of experienced adverse events. The topic list grew more detailed with each interview (Hak, 2004). 
The questionnaires were based on the themes from the interviews and also constructed following the course of events in case of an emergency, adjusted for each of the four provider groups. Thus, the questionnaire for midwifery practices started with contact with the ambulance dispatch centre, followed by cooperation with the ambulance crew, transport to the hospital, arrival at the hospital, and cooperation with hospital staff. Insights from the interviews were used to construct response categories and where relevant open space was added to elicit new responses. Providers were asked how often emergency situations with pregnant or labouring women occurred and how they were experienced, what guidelines were available to cope with such a situation, how responsibilities were allocated to different care providers, how cooperation with other care providers was experienced, and how emergency transfer for women in labour could be improved. The client questionnaire was developed in the same way as the providers' questionnaires, but seen from the clients' perspective. This means: open and closed questions about the emergency they experienced: what happened, who did what, how they reached the hospital, how they were treated, how they felt, how they rated (on a four-point scale) their experiences with different care providers.

\section{Analyses}

The interview data were coded thematically, using the MAXqda software. This means that pieces of text from different interviews on the same topic are given the same code. Some of these codes are derived from literature and are called 'constructed codes', others are based on themes from the interviews and are called 'in-vivo codes'. After coding all the interview sections the codes have been structured in themes and ordered chronologically, according to the process of an obstetric emergency.

Questionnaire data were analysed using STATA 11.0. Only descriptive statistics were used, resulting in frequency distributions and proportions. Written answers to open questions were collected in a text-file and used to select quotes to illustrate the study results.

Throughout this article quotes from the interviews and from client questionnaires are given to illustrate some of the bottlenecks in emergency transfer situations.

\section{Integration}

Structured analysis of the interviews resulted in a list of eight general themes concerning the organisation of emergency transfer in maternity care:

1. Work processes, especially those of the ambulance service and the ambulance dispatch centres.

2. 'Chain of care', contact and responsibilities in case of transfer from one care provider to another.

3. Transport from home to hospital, responsibilities and reasons for time delay.

4. Reception of client at the hospital, availability of delivery room personnel or operating team.

5. Formal protocols or guidelines for interdisciplinary cooperation.

6. Communication between individual care providers, transfer of client information.

7. Cooperation between individual care providers in case of an emergency. 
8. Context factors influencing the transfer, knowledge of each other's competencies, experience.

These general themes were translated into questions for the care providers' surveys. The questions in the surveys were aimed at eliciting feelings of care providers about frequency and severity of emergency transfers, about the process of and experiences with transfer, about cooperation, communication and mutual trust. The results described below are a combination of data from the interviews and the provider surveys. When per centages are mentioned they are derived from one of the provider surveys. When midwives are mentioned, primary care midwives are meant. Otherwise they are called hospital midwives. The emphasis is on highlighting the bottlenecks, as indicated by one or more of the professional groups involved in emergency transfer of maternity care clients.

\section{FINDINGS}

\section{Response}

Four primary care midwives, five ambulance nurses, two operators from an ambulance dispatch centre, four general practitioners, two obstetricians, three hospital midwives, and one obstetric nurse were interviewed, with at least one interviewee from each trauma region. The interviews, held in the autumn of 2009, lasted on average about 40 minutes, were audio-recorded and transcribed verbatim. The response to the survey among professionals was 108 filled-in questionnaires (60\%). Table 1 shows the response rate to these questionnaires. The response from GP out-of-hours cooperatives was low and those who did respond said they seldom or never had experienced an emergence situation concerning a pregnant or labouring woman. Therefore, GP out-of-hours cooperatives are excluded from further analyses.

\section{[TABLE 1]}

The response to the client survey was 42 filled-in questionnaires. By leaving the distribution of the client questionnaires to the midwives we had no control over the inclusion process and therefore could not calculate the response rate. However, we did make an estimation. On the basis of the study by Amelink-Verburg et al. (2008) it was estimated that in a period of six months about 100 clients in 50 midwifery practices (10\% of all midwifery practices) would experience an urgent transfer from home to hospital. This would amount to a maximum response of 90 clients in 45 practices during six months. Therefore the client response rate in this study is estimated to be $47 \%$.

The clients were on average 30 years old and half of them (21) gave birth to their first child. The planned and actual place of birth is shown in Table 2.

\section{[TABLE 2]}

\section{Clients experiences}

The clients described their experiences with the emergency situation, from their own perspective. That means they not always used the 'medical language' we are used to and did not describe their experiences in terms of 'referral' or 'intervention'. Of the 42 women, who all expected to be assisted by their own midwife, 11 (26\%) were referred to a gynaecologist before the infant was born, eight of whom had to be 
transferred from home, the other three were already in the hospital at the time of the referral. Table 3 shows the timing of the transfer to hospital, combined with the way these women were transported. Four women went to the hospital even before labour started. Thirteen women were transported to hospital during labour, nine by ambulance, two by the midwife and two in their own car. Twenty-four women were transported to the hospital immediately after the infant was born, all by ambulance, one woman was transported by ambulance three hours after the infant was born.

\section{[TABLE 3]}

Most clients mentioned some reason for being transferred to the hospital. In 16 of the 42 cases (38\%) excessive blood loss was mentioned, in six cases (14\%) placenta problems were mentioned (with or without excessive blood loss) and a number of other situations were described, ranging from meconium stained fluid or fetal heart rate irregularities to the need for pain relief or a suspicion of a HELLP-syndrome. In total thirty four respondents (81\%) were transported to the hospital by ambulance, including the 16 women with excessive blood loss. For five of these women a second ambulance or other assistance had to be called to help move her.

Looking back at their experiences the majority of these 42 clients are very positive about the care they received. On average $71 \%$ responded very positive on the questions about treatment by the ambulance crew and even more (79\%) responded that they were taken very good care of at the hospital. Fewer women (52\%) answered that it was completely clear to them who to turn to in the hospital when they had questions.

\section{Overall impression}

The overall outcome from the 21 semi-structured interviews, as expressed by most interviewees, was that at the personal level cooperation is often adequate, but mostly informal. The overall conclusion of the care providers, in the interviews as well as in the survey, was that real incidents, with mortality or serious morbidity as result, are rare. Care providers in the study explained that they usually know how to find each other when needed, that cooperation usually goes smoothly, and clients usually receive all the care they need. This is illustrated by the client experience in quote one. Many of the respondents emphasised that knowing each other personally is important in providing good quality care in emergency situations. Quote one: Client:

G3P2 $42^{\circ}$ week of pregnancy. I was scheduled to have an induction that day, but I woke up at night with a lot of blood loss. My midwife had told me I could call her anytime, so I did. She came immediately. The baby's heart rate was fine, but I lost a lot of blood. Because the hospital is close by the midwife and I quickly went to the hospital in 1 car. My partner followed in our own car. There the obstetrician attended us and made an ultrasound. At that moment my water broke en after 2 contractions I gave birth. The amniotic fluid was bloody and there were a number of clots behind the placenta, but our son is born healthy!

Below, the eight general themes as derived from the qualitative analysis and used in the questionnaires will be presented to draw a picture of the chain of events and the experienced bottlenecks in emergency obstetric care in the Netherlands. 


\section{Work processes of ambulance service and ambulance dispatch centre}

Interviewees from the ambulance service indicated that when a midwife calls the ambulance dispatch centre, the operator will almost always accept her assessment of the urgency of the requested transport and send an ambulance accordingly. However, $36 \%$ of the midwives in the survey answered that the operator never tells them how long it will take the ambulance to reach them. Another 33\% of the midwives answered that they only seldom receive a time indication for arrival of the ambulance. Occasionally midwives will ask for an ambulance with incubator, but apparently they do not realise, and are often not told, that this will take extra time, because, for instance, there is only one incubator available in the trauma region and the ambulance will have to go and pick it up first. Some ambulance dispatch centres explained that they will therefore send a second ambulance straight to the client, but that is not standard procedure.

When a client herself calls for an ambulance the dispatch centre operator, according to the interviewees, has to ask the client for the name and telephone number of her midwife, because that information is not readily available at the ambulance dispatch centre. As the gynaecologist in quote two said, a call from a client may lead to misunderstandings when the operator does not suspect any relation with pregnancy or childbirth.

Quote two: Obstetrician:

When a client calls 112 with abdominal pain and does not tell the operator she is 38 weeks pregnant, then the operator will probably advise her to go to a GP out-of-hours cooperative. I can understand that, but that is miscommunication.

Ambulance personnel and dispatch centre operators, as in quote three, expressed the feeling that midwives may be too reluctant to call them in case of an emergency and do not realise that, even after calling an ambulance, it may take considerable time before the client will arrive in a hospital.

Quote three: Dispatch centre operator:

In my view, as soon as you feel something may be amiss..........in my view that is an indication to call an ambulance.

\section{Chain of care}

The organisation of maternity care in the Netherlands is such that the 'chain of care' in case of (emergency) transfer of a maternity client almost always starts with the midwife, because midwives are the primary care providers for pregnant women. When complications (threaten to) arise the midwife will refer her client to an obstetrician in a nearby hospital. In urgent cases she will call an ambulance to transport the patient. Midwives as well as labour ward staff described that contact between midwife and hospital is usually by telephone and additional client information is often on paper, because electronic systems in primary care and in hospitals do not match. The majority of survey respondents (more than $90 \%$ in each group) agree that the transfer of information usually or always goes smoothly, because they know each other, as is illustrated in quote four.

Quote four: Midwife:

In this hospital, this region, when you know who you are talking to, then you know it will be all right. But if I had to call another hospital, in a different situation, I would ask: do I have to consult with the gynaecologist as well or is this enough? 
However, they add that this process may lead to the loss of vital information, especially when that information has to be retold more than once, by different people: from midwife to ambulance nurse and/or obstetric nurse, from nurse to hospital midwife or resident, from hospital midwife or resident to obstetrician. Less than half of the midwives (44\%) in the survey said they can usually speak to the obstetrician (on call) directly, but that obstetrician may not be present at the hospital at that moment. This is illustrated by the experience of the client in quote five. Quote five: Client:

Gave birth at 2.33 a.m., placenta was stuck; 3.03 a.m. midwife called an ambulance $\rightarrow$ arrival 3.20 a.m., they couldn't take me downstairs lying, so the fire brigade was called $\rightarrow$ arrival \pm 4.15 a.m., we went to the hospital, on arrival the anaesthesiologist and the obstetrician were not present yet.

\section{Transport}

Respondents were asked to indicate how often in their experience an emergency transfer occurred. Table 4 provides an overview of the answers. With this question (as with most others) we were not looking for actual numbers, but for the impression of different care providers of the frequency of emergency situations they encountered, because that may influence their inclination to support changes in the organisation of emergency transfers.

\section{[TABLE 4]}

It is no surprise that labour ward staff more often experience an emergency transfer than primary care midwifery practices, because in general more than one and often four or five midwifery practices refer their patients to the same hospital.

To learn more about the frequency of ambulance transport for maternity clients, the ambulance services were asked to provide registration data about the number of times they transported a maternity care client in the previous year. Thirteen of the eighteen responding ambulance services could provide the data and based on that we calculated that $0.88 \%$ of high urgency transports and $1.18 \%$ of low urgency transports, making an average of $0.98 \%$ of all transports were related to pregnancy and childbirth. This illustrates the reported feeling of most of the ambulance personnel that they seldom are confronted with obstetric emergencies, as does the remark from one of the ambulance nurses in quote six.

Quote six: Ambulance nurse:

Maternity care is really not very prominent in emergency care awareness To the question what additional factors in general, besides the medical condition of the woman and her (unborn) infant, made them classify a referral as urgent, midwives mentioned eight factors, of which the most prominent were on one hand: (1) the length of the pregnancy, (2) the duration of labour, and (3) the parity of the client, and on the other hand (4) the amount of traffic and (5) the distance to the nearest hospital. Four of these five factors (excluding factor four), combined with the factor 'time of day (daytime or evening/night/weekend)' are found to be the most pressing reasons for midwives to call for an ambulance, and not let the client go to the hospital with her own transport or take her to the hospital in the car of the midwife. 
A home birth usually takes place in the bedroom, which is often an upstairs room. In case of an emergency additional assistance may be needed from a second ambulance crew or the fire brigade to carry the woman downstairs. This may lead to time delay, when the first ambulance crew has to wait for the assistance to arrive before being able to help the woman. A small majority of midwives in the survey (53\%) and a large majority of ambulance nurses (89\%) had experienced this situation at least once. Sometimes midwives anticipate this by asking women to stay on the ground floor or helping them downstairs before the ambulance arrives and sometimes the dispatch centre will send a second ambulance along with the first. In Amsterdam, for instance, the operator at the dispatch centre will ask where the patient is and will send the fire brigade along with the ambulance whenever the woman is not on the ground floor in a building without an elevator. On occasion their help will not be necessary, but the basic thought is: better available and not needed than not available when needed.

\section{Reception at the hospital}

Maternity care clients, even if they arrive by ambulance, will usually not be admitted to the Emergency Room, but will be brought to the delivery rooms directly. In some hospitals that means a different entrance, which may cause some time delay, especially when the ambulance crew is not familiar with that hospital. On average, clients brought in by ambulance, are seen as more urgent than clients brought in by midwives, according to the labour ward staff. Most midwives (83\%) in the survey said they would go to the hospital themselves when their client was transported by ambulance, but only in special circumstances would they accompany her in the ambulance, and only at the request of the ambulance crew, for instance when the birth is imminent.

In most hospitals obstetricians and anaesthesiologists are on call outside office hours, which often means they are not present in the hospital, but must be called in. The first assessment of the client on arrival at the hospital will then be by a resident or a hospital midwife, who, in turn, will decide whether or not to notify the obstetrician immediately. This procedure in itself may lead to time delay, but the midwife may also contribute to that by not being explicit about the urgency of her call. Labour ward staff in the survey said they were not always aware of the urgency of a referral after a call from a midwife saying she had send a client to the hospital. And they almost unanimously would like to be notified of the expected arrival of the ambulance, so they could properly prepare. However, only one in four midwives and one in three ambulance nurses reported that they usually or always call the hospital to let them know the ambulance is on its way.

An additional problem, not often mentioned by the professionals, but strongly illustrated by quote seven from a client, is the occasional unavailability of a delivery room.

Quote seven: Client:

Reason for hospitalisation was insufficient dilatation (stayed at $91 / 2$ centimetres).

Ambulance was quickly on the spot. However, the problem was that in the hospitals that were closest by no delivery room was available. Midwife had to call 5 hospitals in 3 different provinces. Finally, the last one made room for us! 


\section{Formal protocols or guidelines}

Ambulance services work on the basis of protocols for each conceivable situation, so they also have a protocol for obstetric emergencies (Ariëns et al., 2009). In the Netherlands emergency operators of the ambulance dispatch centre use a national triage protocol to assess the urgency of the call and to collect information about the patient. All respondents from the ambulance services confirmed this.

Midwives, however, indicated they have no protocols for emergency situations, and not all of them are familiar with the ambulance protocols for emergency obstetric situations. The general opinion of the respondents is that this may lead to misunderstandings about who is responsible for what action in an emergency situation. Midwives indicated they also have no protocols for calling an ambulance dispatch centre or for the information they need to provide to the operator in order to get an ambulance as quickly as possible. The majority of the responding midwives (55\%) would welcome such a protocol. Guidelines for referral from a primary care midwife to an obstetrician and back are available and widely used (CVZ, 2003). Guidelines or protocols about home birth, for instance the suitability of the house, or the Body Mass Index of the woman, are not formalised but midwifery practices devise their own policies concerning these issues.

\section{Communication}

Most midwives participate in formal, regular meetings with other maternity care providers: obstetricians, paediatricians, hospital midwives, obstetric nurses, general practitioners and maternity care assistant organisations, in local maternity care consultation and cooperation groups (in Dutch: Verloskundige

SamenwerkingsVerbanden or: VSVs). Ambulance services and ambulance dispatch centres are not included in these VSVs. Respondents indicate that informal meetings between midwives, obstetricians and labour ward staff occur regularly when a client is referred, with or without urgency, or when a client chooses to give birth in hospital, assisted by her own midwife, which will improve cooperation and communication, as expressed in quote eight.

Quote eight: Midwife:

...we are increasingly cooperating with one particular hospital, you can tell that people there are starting to get to know you and therefore treat you differently. Formal meetings between midwives and ambulance crews or between hospital staff and ambulance crews are said to be rare.

\section{Cooperation}

All respondents agree that cooperation is needed as soon as the ambulance arrives. According to the ambulance nurses the first assessment will be whether the client should be transported immediately or needs to be stabilised first. Misunderstanding is mentioned by both professionals as a possibility when the ambulance crew expect the midwife to have already started an intravenous drip, while often the midwife feels she is insufficiently trained to do so. Moreover, during the minutes between calling the ambulance and its arrival the midwife is otherwise occupied with her client, as is illustrated in quote nine.

Quote nine: Midwife:

I am not skilled in starting a drip. And I think I am more than busy during the fifteen minutes before the ambulance arrives, to be able to do that as well. 
But all interviewees and survey respondents emphasise that in an actual emergency situation cooperation usually goes smoothly, especially when people know each other. The same holds for cooperation between midwives and labour ward staff, the better people know each other, the better the cooperation in emergency situations will be.

\section{Context factors}

Context factors are factors that can influence the cooperation in emergency situations but are not strictly related to one of the above presented themes. For instance, knowledge about each other's competencies and experience will influence the cooperation. Respondents agree that when care providers are more familiar with each other's work routines, they will know better what to expect and will be better able to anticipate the other's expectations. The same holds for experience: more experienced care providers know better what to expect and how to co-operate.

Quote 10: Client:

After the birth of my baby the placenta would not detach completely. I lost blood. The ambulance arrived, a drip was started. My experience is that the ambulance personnel did not realise the severity of the situation. They needed to be constantly given directions by the midwife!!

A different context factor is the way in which emergency situations are being evaluated by care providers, especially in a multidisciplinary setting. Most respondents indicated that incidents will be evaluated within each professional group, but ambulance services usually do not give feedback to midwives and midwives not to ambulance services. Evaluation of emergency situations by midwives and hospital staff happens more often, because they see each other on a regular basis.

\section{DISCUSSION}

In general, the majority of respondents, interviewees as well as survey respondents, were positive about the organisation and quality of the emergency care for maternity clients. The general attitude is that the lines of communication are clear, the responsiveness is high, and calamities are very rare. Cooperation is not seen as a problem, especially in situations where care providers know and trust each other. Nevertheless, several issues or bottlenecks have been described that could be improved. On the basis of these bottlenecks a number of recommendations have been formulated.

The first recommended improvement, not because it is the most important, but because it affects the first action to be taken in case of an emergency, would be a protocol for midwives about calling an ambulance dispatch centre and providing the necessary information to the emergency operator. This protocol would have to include items about the expected time of arrival of the ambulance and about the reasons to request a second ambulance or an ambulance with incubator.

A second recommended improvement would be to introduce multidisciplinary training sessions for emergency situations in all trauma regions. The results of this study showed that many of the respondents were not familiar with the guidelines, protocols and work processes of care providers from other professions. By training together, especially within their own region, different care providers not only get to know each other's protocols, but also each other. This will improve communication and cooperation (Freeth et al., 2009 and Van et al., 2010). In some trauma regions 
multidisciplinary training has already been introduced and the preliminary results are positive (Baerts et al., 2009).

A third recommended improvement relates to the referral process from midwife to obstetrician. Sometimes a time delay is caused by the wish of the receiving care providers at the hospital to make a new assessment of the urgency of the situation, before calling in the obstetrician. A referral protocol, in which the midwife clearly states her assessment, which is not contradicted by the hospital staff, might be an improvement.

In a recent study by Evers et al. (2010) about perinatal mortality and severe morbidity the conclusion was that: 'Infants of pregnant women at low risk whose labour started in primary care under the supervision of a midwife in the Netherlands had a higher risk of childbirth related perinatal death and the same risk of admission to the NICU compared with infants of pregnant women at high risk whose labour

started in secondary care under the supervision of an obstetrician.' In the discussion the authors suggest that the Dutch system based on risk selection, referral, and transfer may not be as effective as once thought but instead may contribute to the high perinatal mortality. They argue that the system itself may lead to delay. Firstly, delay in diagnosing complications in primary care, because a midwife is not constantly present during the first stage of labour and the fetal heart rate is not monitored continuously in primary care. Secondly, delay in treatment, because of the necessary transport in case of a planned home birth. And thirdly, delay in interventions because obstetricians in secondary care may underestimate the problems of a woman who is referred by a primary care midwife. In addition, they conclude, essential information can be lost during the referral. These conclusions about the causes for possible delay corroborate the findings of our study. They emphasise the importance of a perfectly functioning organisation of emergency transfer in maternity care and the serious consequences that can occur when one or more links in the chain fail to perform.

Two of the reasons Davis-Floyd (2003) described in her paper about transport stories for a birth to go awry were that the midwife may wait too long to summon transport, or that the hospital staff taking the call may not understand the urgency of the problems. These reasons are similar to some of the bottlenecks described in this study. One of the solutions a midwife in one of those transport stories came up with was to develop a network of relationships with individuals in the hospitals. That also fits the recommendations from this study. More in general, the recommendations of Davis-Floyd were to invest in a dialogue and in the relationship between the hospital staff and the midwives in order to establish mutual trust and smooth cooperation well in advance of any emergency situations. So, regardless of the very different conditions and circumstances concerning home birth in the USA and Mexico on the one hand and the Netherlands on the other hand emergency transport in maternity care may face some of the same problems and may need comparable solutions. Our research has also shown that, although guidelines and protocols are regarded as necessary to ensure timely and appropriate responses, personal relationships are at least as important for a smooth and continuous care process. Transfer of care from one care provider to another during labour or birth is not unusual in the Dutch maternity care system. It is of vital importance for care providers involved in this transfer, to know and trust each other, to be able to give an adequate reaction when 
needed. Most recommendations therefore are aimed at improving knowledge about each other, for instance by providing combined courses for emergency obstetrics, preferably at the regional level, so people who actually may work together can train together.

\section{LIMITATIONS}

There were limitations to this study. First, the response from GPs in out-of-hours cooperatives was very low, so no conclusions could be drawn from their answers. However, from the four GPs that were interviewed and the seven GPs that did return the questionnaire we learned that their involvement with emergency transfer for pregnant or labouring women is so rare, they could not answer most of the questions. Second, we were unable to calculate an accurate response rate for the client questionnaires, because we lacked information on how many questionnaires were handed out. However, the client questionnaires were not meant to present a representative picture of clients needing emergency transfer, but to illustrate bottlenecks in the organisation of emergency transfer from the clients' perspective.

\section{CONCLUSION}

Primary care midwives, ambulance personnel, labour ward staff, general practitioners and clients participated in the study. The overall impression from the interviews as well as from the questionnaires is that at the personal level cooperation is often adequate, but mostly informal. We have shown that care providers from different professions are not always aware of existing agreements and protocols, are unsure about each other's competencies and do not always know what to expect from the other care provider. This may lead to misunderstandings and time delay, which may negatively affect the client and/or her infant. An emergency transfer from home to hospital during labour or birth can be a traumatic experience. Care providers will have to do everything to make this transfer as smooth as possible. Although each profession has its own rules and protocols, the integration of these into true integrated care is often still lacking and needs to be addressed by all care providers involved.

\section{COMPETING INTEREST}

The authors declare there are no competing interests.

\section{ETHICAL APPROVAL}

Ethical approval for this type of study is not required in the Netherlands.

\section{AUTHORS' CONTRIBUTION}

TW designed the study, contributed to the analyses, drafted and revised the manuscript. JdB organised the data collection and was responsible for the analyses, organised the conference, and contributed to the development of the manuscript and its revision.

\section{ACKNOWLEDGEMENTS}

This study was funded by ZonMw, the Dutch Organisation for Health Research and Development (Grant no. 82711003). We thank Anke Oostveen (KNOV), Ed Ariëns 
Wiegers, T.A., Borst, J. de. Organisation of emergency transfer in maternity care in the Netherlands. Midwifery: 2013, 29(8), 973-980

and Wim ten Wolde (AZN) for their contribution to the design of the study and their continuous support. We also thank all the care providers and clients who participated in the study.

\section{REFERENCES}

Ambulancezorg Nederland, 2010. Ambulancezorg Nederland, 2010. 'Ambulance services in 2009' highlights. 〈http://www.ambulancezorg.nl/download/downloads/80/summaryambulances-in-sight-2009.pdf $\rangle$.

Amelink-Verburg et al., 2008 M.P. Amelink-Verburg, S.P. Verloove-Vanhorick, R.M.A. Hakkenberg, I.M.E. Veldhuijzen, J. Bennebroek Gravenhorst, S.E. Buitendijk Evaluation of 280,000 cases in Dutch midwifery practices: a descriptive study British Journal of

Obstetrics and Gynaecology, 115 (2008), pp. 570-578

Ariëns and ten Wolde, 2009 Ariëns, E.A.R., ten Wolde, W.L.M., (red), 2009. Landelijk protocol ambulancezorg, Herziene versie 7.1 [National Protocol Ambulance Care, revision 7.1]. Zwolle, Ambulancezorg Nederland.

Baerts et al., 2009 A. Baerts, E. Witzier, N. Hagen, H. Houtsmuller, C.van Meir, E. Hallensleben De tweespalt aan de kant. Gynaecologen en vroedvrouwen buigen zich samen over zwangerenzorg [The discord aside. Gynaecologists and midwives work together in the care for pregnant women]. Medisch Contact, 64 (2009), pp. 746-748

CBS, 2009 CBS, 2009. Geboorte naar diverse kenmerken [Birth; key figures] Centraal Bureau voor de Statistiek [Statistics Netherlands].

CVZ, 2003 CVZ, 2003. Verloskundig vademecum 2003. College voor zorgverzekeringen, Diemen.

Davis-Floyd, 2003 R. Davis-Floyd Home-birth emergencies in the US and Mexico: the trouble with transport Social Science and Medicine, 56 (2003), pp. 1911-1931

Euro-Peristat, Scpe, Eurocat, Euroneostat, 2008 Euro-Peristat, Scpe, Eurocat, Euroneostat, 2008. European Perinatal Health Report.

Evers et al., 2010 et al.Perinatal mortality and severe morbidity in low and high risk term pregnancies in the Netherlands: prospective cohort study British Medical Journal, 341 (2010), p. c5639 http://dx.doi.org.proxy.library.uu.nl/10.1136/bmj.c5639

Freeth et al., 2009 et al.Multidisciplinary obstetric simulated emergency scenarios (MOSES): promoting patient safety in obstetrics with teamwork-focused interprofessional simulations Journal of Continental Education Health Professor (The Journal of continuing edudation in the health professions), 29 (2009), pp. 98-104

http://dx.doi.org.proxy.library.uu.nl/10.1002/chp.20018

Hak, 2004 T. Hak Data collection in qualitative research Huisarts en Wetenschap, 47 (2004), pp. 502-508 [in Dutch]

Johnson and Daviss, 2001 Johnson, K.C., Daviss, B.A., 2001. Results of the CPM Statistics Project 2000: a prospective study of births by certified professional midwives in North America. Abstract presented at the annual meeting of the American Public Health Association, Atlanta, GA. M.

Johnson and Daviss, 2005 K.C. Johnson, B.A. Daviss Outcomes of planned home births with certified professional midwives: large prospective study in North America British Medical Journal, 330 (2005), p. 1416

MAXqda, 2001 MAXqda, 2001. Reference Manual for the Text Analysis Software MAX Qualitative Data Analysis 10 for Windows 2000, XP, Vista, and 7. VERBI Software. Sozialforschung. GmbH Marburg/Germany.

Meiberg, A.E., IJzermans, C.J., Bakker, D.H.de., 2007. Samenwerking in de katen van acute zorg: een zelf-evaluatie-instrument. [Cooperation in emergency care: a self-evaluationinstrument] NIVEL, Utrecht. 
Offerhaus and van der Hoff, 2008 Offerhaus, P., van der Hoff, I., 2008. Verloskundige zorg, veilige zorg! 2008-2012. 5-jaren plan Patiëntveiligheid. [Maternity care, safe care! 20082012 5-year Plan on Patient safety]. Utrecht, KNOV.

PRN, 2011 PRN, 2011. Perinatale Zorg in Nederland 2008/Perinatal Care in the Netherlands 2008. Stichting Perinatale Registratie Nederland. Utrecht, Stichting Perinatale Registratie Nederland.

Van et al., 2010 Ven J. van de, Houterman S., Steinweg R.A.J.Q., et al., 2010. BMC Pregnancy and Childbirth 10, 59, http://www.sciencedirect.com.proxy.library.uu.nl/science?_ob=RedirectURL\&_method=exte rnObjLink\&_locator $=u r l \& \_i s s n=02666138 \&$ origin $=$ article $\& \_z o n e=a r t$ page\&_plusSign $=\% 2$ B\& targetURL=http\%253A\%252F\%252Fwww.biomedcentral.com\%252F14712393\%252F10\%252F59

\section{FIGURE AND TABLES}

Fig. 1. Proportion ofwomeninmaternitycarebycaregiveratdifferentstages, referrals duringantenatalandnatalcare, andactualplaceofbirth2008. Source: PRN,CBS.

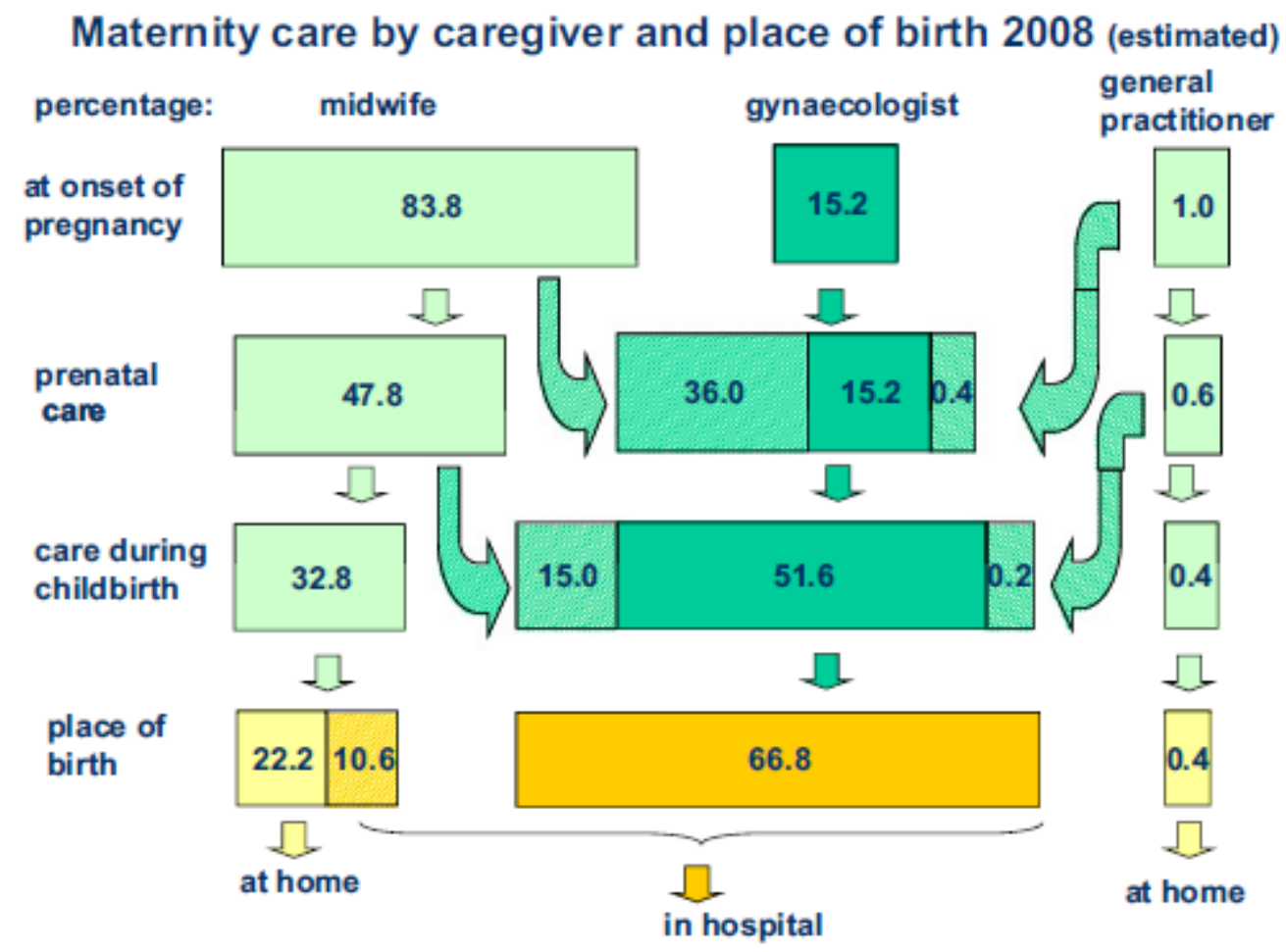


Table 1

Care providers survey.

\begin{tabular}{lccc}
\hline & $\begin{array}{c}\text { Invited } \\
\text { n }\end{array}$ & $\begin{array}{c}\text { Response } \\
\text { n }\end{array}$ & $\begin{array}{c}\text { Response } \\
\%\end{array}$ \\
\hline Primary care (community) midwives & 88 & 53 & 60 \\
Labour ward staff $^{\dagger}$ & 44 & 30 & 68 \\
Ambulance services & 25 & 18 & 72 \\
GP-out-of-hours cooperatives & 23 & 7 & 30 \\
Total & 180 & 108 & 60 \\
\hline
\end{tabular}

* Response: 11 gynaecologists, eight hospital midwives, two obstetric nurses, and nine not specified.

$\uparrow 17$ Ambulance nurses and one not specified.

Table 2

Planned and actual place of birth.

\begin{tabular}{|c|c|c|c|c|}
\hline Actual/planned & $\begin{array}{c}\text { At } \\
\text { home }\end{array}$ & $\begin{array}{l}\text { In hospital with } \\
\text { own midwife }\end{array}$ & $\begin{array}{l}\text { In hospital with } \\
\text { obstetrician }\end{array}$ & Total \\
\hline At home & 16 & 1 & 8 & 25 \\
\hline In a birth centre & 1 & 0 & 0 & 1 \\
\hline $\begin{array}{l}\text { In hospital with own } \\
\text { midwife }\end{array}$ & 10 & 3 & 3 & 16 \\
\hline Total & 27 & 4 & 11 & 42 \\
\hline
\end{tabular}

Table 3

Timing and way of transfer to hospital.

\begin{tabular}{lcccc}
\hline \multirow{2}{*}{ Timing of transfer } & \multicolumn{3}{l}{ Way of transfer } & \\
\cline { 2 - 5 } & Own car & Midwife's car & Ambulance & Total \\
\hline Before start labour & 4 & 0 & 0 & 4 \\
During labour & 2 & 2 & 9 & 13 \\
Immediately after birth & 0 & 0 & 24 & 24 \\
Hours after the birth & 0 & 0 & 1 & 1 \\
Total & 6 & 2 & 34 & 42 \\
\hline
\end{tabular}


Wiegers, T.A., Borst, J. de. Organisation of emergency transfer in maternity care in the Netherlands. Midwifery: 2013, 29(8), 973-980

Table 4

Experienced frequency of emergency transfers.

\begin{tabular}{|c|c|c|c|c|c|c|}
\hline & \multicolumn{2}{|c|}{$\begin{array}{c}\text { Primary care } \\
\text { (community) } \\
\text { midwives }\end{array}$} & \multicolumn{2}{|c|}{$\begin{array}{c}\text { Labour } \\
\text { ward staff }^{\dagger}\end{array}$} & \multicolumn{2}{|c|}{$\begin{array}{c}\text { Ambulance } \\
\text { services }\end{array}$} \\
\hline & $\mathrm{n}$ & $\%$ & $\mathrm{n}$ & $\%$ & $\mathrm{n}$ & $\%$ \\
\hline More than once a week & 1 & 2 & 11 & 44 & 2 & 11 \\
\hline Once a week & 5 & 10 & 7 & 28 & 0 & - \\
\hline More than once a month & 15 & 29 & 3 & 12 & 7 & 39 \\
\hline Once a month & 11 & 22 & 2 & 8 & 6 & 33 \\
\hline Once a year & 19 & 37 & 2 & 8 & 3 & 17 \\
\hline Total & 51 & 100 & 25 & 100 & 18 & 100 \\
\hline
\end{tabular}

* Two missing.

${ }^{\dagger}$ Five missing. 